Volume 9 No.6, June 2020

International Journal of Advances in Computer Science and Technology

Available Online at http://www.warse.org/IJACST/static/pdf/file/ijacst06962020.pdf

https://doi.org/10.30534/ijacst/2020/06962020

\title{
Deep Learning based Pneumonia detection using X-Ray
}

\author{
Asaph M Joyer George ${ }^{1}$, Akshay V Anil ${ }^{2}$, Abhishek B ${ }^{3}$, Gayathri R Krishna ${ }^{4}$ \\ ${ }^{1}$ Mangalam College of Engineering, Kottayam, India, asaph1390@ gmail.com \\ ${ }^{2}$ Mangalam College of Engineering, Kottayam, India, akshayvanil117@gmail.com \\ ${ }^{3}$ Mangalam College of Engineering, Kottayam, India, abhishek.babu3@ gmail.com \\ ${ }^{4}$ Mangalam College of Engineering, Kottayam, India, gayathri.krishna@mangalam.in
}

\begin{abstract}
Pneumonia has been one of the diseases that affect the lungs and within a short period of time, due to the accumulation of fluid in lungs patients may experience shortage of breath resulting in death. So the early diagnosis is important for helping the patients. This paper focuses on the detection of pneumonia by x-ray imaging and using a model trained by using convolutional neural networks. In this paper the deep learning architecture for the classification task, which is trained with modified images, through multiple steps of preprocessing? Our classification method uses convolutional neural networks for classifying the images. The trained model yields an accuracy of $96 \%$.
\end{abstract}

Key words: Neural Network, Pneumonia, X-ray Imaging, Automation, Detection

\section{INTRODUCTION}

Pneumonia is an infection of the lungs that may be caused by bacteria and viruses. The infection causes the lung's air sacs to become inflamed and fill up with fluid. This causes the patient to drown. The symptoms of pneumonia can range from mild cough to fever, chills, and trouble breathing .People over age 65 are at risk because their immune system is becoming less able to fight off infection. Infants and children two years of age are also at increased risk because their immune systems are not yet fully developed. Chest X-ray helps the doctor diagnose pneumonia and determine the extent and location of the infection, but it can't tell your doctor what kind of germ is causing the pneumonia infection.

Recent Improvements in the CNN architectures and deep learning approaches has made new possibilities to detect the infections early that are caused by pneumonia. Deep learning still cannot replace doctors in diagnosis but it can provide support for experts in the medical field in performing time-consuming works, such as examining chest X-ray for pneumonia. In this paper we present a deep learning architecture tailored to pneumonia diagnosis. With this approach we reduce the computational and memory requirement significantly, without sacrificing the classification performance therefore increasing accuracy to $96 \%$.

\section{LITERATURE SURVEY}

In this paper the network starts off with some random weights and zero bias which are the "parameters" which will be influencing the neural network's decision on whether an image is one of a pneumonia scan or not. Once the input data is processed through ANN using these weights and biases, it will use activation function which will return either a 0 or 1.Here a loss function to calculate the margin of error on prediction is solved by back propagation which adjusts the network's weights and biases to make better predictions with an accuracy of $80 \%$ [1].

Early diagnosis and treatment of pneumonia is critical to preventing complications including death. With approximately 2 billion procedures per year, chest X-rays are the most common imaging examination tool used in practice, critical for 
screening, diagnosis, and management of a variety of diseases including pneumonia. However, two thirds of the global population lacks access to radiology diagnostics, according to an estimate by the World Health Organization, There is a shortage of experts who can interpret X-rays, even when imaging equipment is available, leading to increased mortality from treatable diseases. Here the authors develop an algorithm which detects pneumonia from frontalview chest $\mathrm{X}$-ray images at a level exceeding practicing radiologists [2].

Standardized methods for electronic auscultation and chest ultrasound and compare their utility for detection of pneumonia to standard approaches. Utilizing signal processing techniques, the authors aim to characterize lung sounds and through machine learning, develop a classification system to distinguish pathologic sounds. Data will allow a better understanding of the benefits and limitations of novel diagnostic techniques in pediatric pneumonia [3].

A deep convolutional neural network model with transfer learning has been proposed. Before passing images to model, preprocessing is done using filtering, gamma correction, equalization, and compression methods. The proposed model is compared with ResNet, ImageNet, Xception, and Inception in terms of precision, recall, accuracy, and ROC accuracy score [4].

After training the model for 500 epochs, test time augmentation was done and the model was tested for performance on the test data. The model accomplished an accuracy of $92.9 \%$, and is considerably superior to the baseline accuracy of $63 \%$. The precision and recall values were approximately around 0.9088 and 0.9927 respectively. These values prove that the model is well-fit. After training and building the model, the weights are saved into a pytorch file for prediction of new single image .The pre-trained model is then loaded and evaluated based on the architecture of the trained model [5].

\section{PROPOSED WORK}

Here the transfer learning, using the first 16 layers of a pre-trained Oxford VGG19 Network, to identify the image classes. The convolutional neural network models (CNN) developed by the VGG won the image classification tasks. This is perfect for image classification with our data. The stages for the classification are divided into a number of stages. It loads the VGG model and uses the Keras deep learning library. Keras provides an interface for using pre-trained models that reduces complexity.

\section{The Pre-Processing Stage}

The images preprocessed to enhance their quality using filtering methods. The noise removal process gives enhanced images, which were used to find the ROI of images using segmentation. During the cauterization process of $\mathrm{x}$-ray images there is the possibility of noise added with the images. So it is necessary to remove the noise using the various Noise Removing Techniques.

\section{The Feature-Extraction Stage}

The purpose of feature extraction in this is to convert a two-dimensional image into a feature vector, which can be further utilized as the input to the phase of the classifier. The extracted features will provide the characteristics of the input type to the classifier by using the description of the required properties of the image into feature vectors.

\section{The Classification Stage}

The trained model classifies the preprocessed images based on the features that are most relevant to being affected by pneumonia. The model is a binary classifier that classifies to either 0 or 1.1 for pneumonia and 0 being not affected by pneumonia.

\section{SYSTEM ARCHITECTURE}

The proposed model uses Convolution neural network for the identification of pneumonia using Xray radiograph. The Convolution neural network works like our brain in the context of image recognition. This model contains a dataset of 5863 images from the Kaggle dataset. This dataset undergoes several processes as given below.

Step 1: Convolution

Step 2: Rectifier Linear Unit Layer

Step 3: Pooling

Step 4: Flattening

Step 5: Full Connection 


\section{Convolution}

This is the first step; it will act as a filter. The output of this process is feature maps and this process includes learning the parameters of such maps and pattern detection. The advantage of this operation is that it reduces the size of the input image. The output we get has fewer cells than the original input image. However, the role of the feature detector is to convert the information in the input image and filter the parts that are integral to it and exclude the rest. In this case we convert the raw $\mathrm{x}$-ray radiograph into a cluster of feature maps which helps set the parameters needed for detection of pneumonia and also reduce the size of $\mathrm{x}$-ray radiographs.

\section{Rectifier Linear Unit Layer}

The rectifier function is to increase the non-linearity in the x-ray images because the images are naturally non-linear. In every $x$-ray image there are non-linear features like the borders, noise, transition between pixels, etc. The rectifier serves to break up the linearity even further in order to make up for the linearity that we might impose an image when we put it through the convolution operation.

\section{Pooling}

The pooling function is used to enable the convolutional neural network to detect the particular pattern when presented in any manner. If our X-ray image is posing in a different angle and in different depth, a pooling function is used to detect the pattern of x-ray. Pooling is used to recognize that particular pattern from all of these different patterns that are possible. The network needs to acquire a property that is known as "spatial variance." This property makes the network capable of detecting the object in the image without being confused by the differences in the image's textures.

\section{Flattening}

The output of the pooling stage is a matrix and is needed to be converted into a single column. In this stage the breakdown of the dataset to how we move from pooled to flatten layers when working with CNN. Flattening involves transforming the entire pooled feature map of the dataset into a matrix of single columns.

\section{Full Connection}

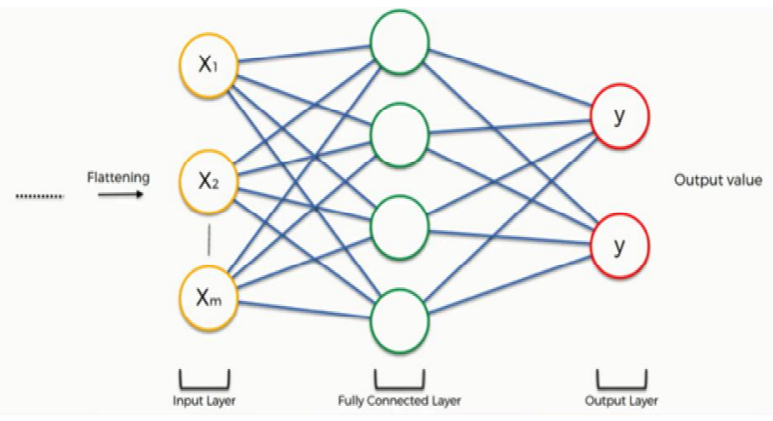

Figure 1: Full connection

This is the final part in which everything that has been done in the previous step will be merged together. By combining the input to the ANN we get to envision a fuller picture of how convolutional Neural Networks operate and how the "neurons" that are finally produced learn from the classification of images.

\section{RESULT}

The Results of this trained model with the whole database containing different variations of x-ray radiograph yields an accuracy of $96 \%$. As convolutional networks are able to learn features when trained on larger datasets the results achieved when trained with only one variant of images will not be used. After the parameters of the network are set, an overall accuracy of $96 \%$ was achieved. Furthermore, the trained model was tested on each validation set image. This test was performed on every image from the kaggle dataset. The complete procedure was described respectively from collecting the images used for training and validation to image.

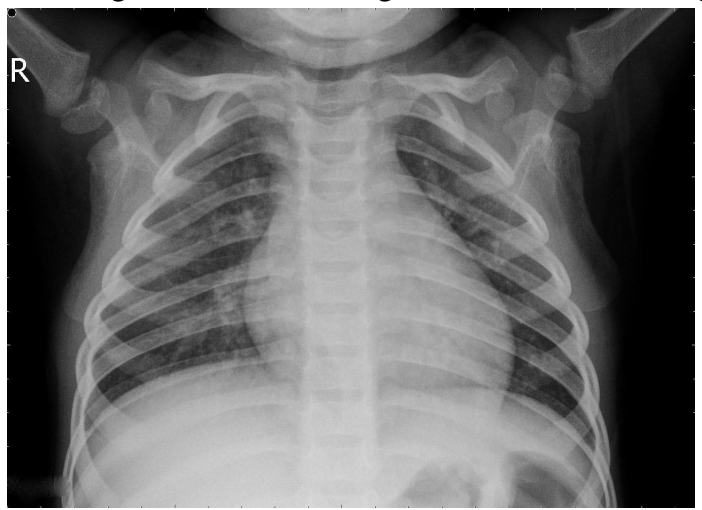

Figure 2: Normal X-ray 


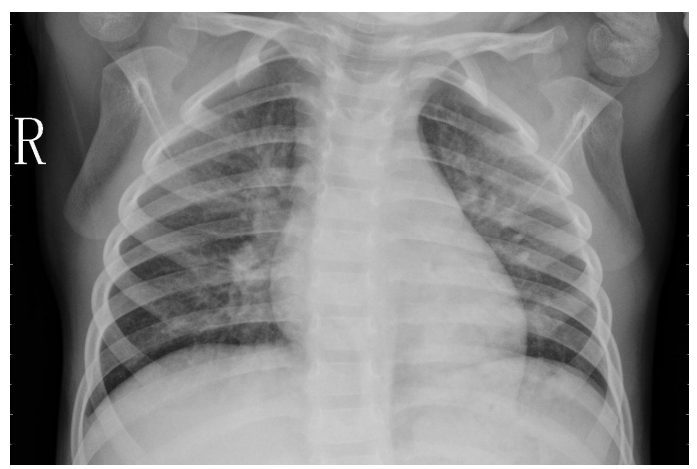

Figure 3: Pneumonia affected X-ray

The model is converging in the graph which shows the decrease in loss and validation loss with epochs.It is also able to reach $96 \%$ validation accuracy in just 10 epochs.
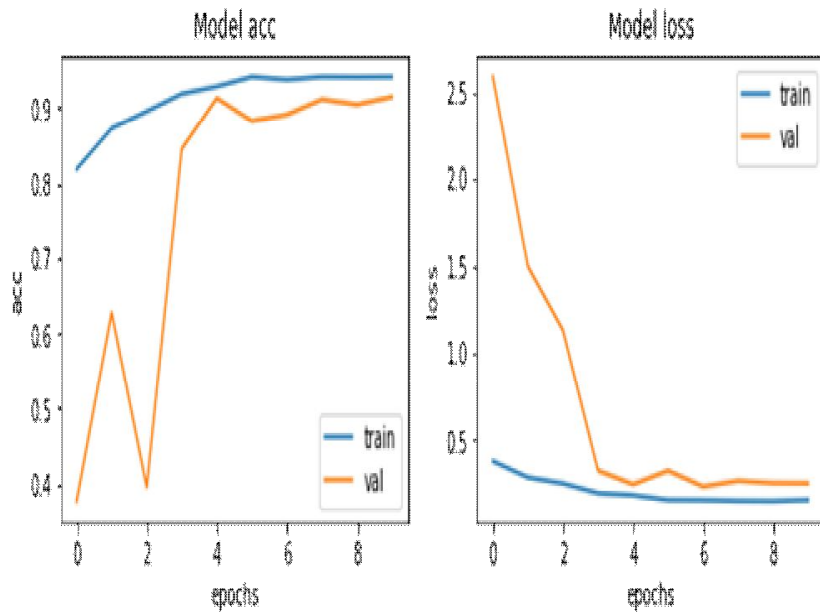

Figure 4: Model accuracy and Model loss

\section{CONCLUSION}

Pneumonia is a severe acute respiratory condition that leads to death within a short period of time as we know in the case of covid-19, the above proposed method will help doctors to predict pneumonia in optimal time with high accuracy and it will help the patient in getting proper medication before the condition of the lungs become worse. The model can be further improved by adding more images to the dataset.
As this project is far from complete to cope with the real world problems, the incorporation of different types or elements, showing variation or variety may be used to improve the model.

\section{ACKNOWLEDGEMENT}

The authors wish to thank Principal Manoj George, Dr. Vinod P Vijayan H.O.D, Computer Science Department, and Mrs. Gayathri R Krishna for the proper guidance, valuable support, and helpful comments during the proofreading.

\section{REFERENCES}

1. Zhou, Bolei, Khosla, Aditya, Lapedriza, Agata, Oliva, Aude, and Torralba, Antonio, "Learning deep features for discriminative localization" IEEE Conference on Computer Vision and Pattern Recognition, pp. 2921-2929, 2016.

2. P. Rajpurkar et al., "Chexnet: Radiologist-level pneumonia detection on chest $x$-rays with deep learning", 2017.

3. Z. Kermany Daniel, Kang and Goldbaum Michael, "Labeled Optical Coherence Tomography (OCT) and Chest X-Ray Images for Classification", 2018.

4. O. Russakovsky et al., "Imagenet large scale visual recognition challenge", International Journal of Computer Vision, vol. 115, no. 3, pp. 211-252, 2015

5. G. Litjens et al., "A survey on deep learning in medical image analysis", Medical image analysis, vol. 42, pp. 60-88, 2017. 